\title{
Disordered and densely packed ITO nanorods as an excellent lithography- free optical solar reflector metasurface for the radiative cooling of spacecraft
}

Yildirim, Deniz Umut, Ghobadi, Amir, Soydan, Mahmut Can, Atesal, Okan, Toprak, Ahmet, et al.

Deniz Umut Yildirim, Amir Ghobadi, Mahmut Can Soydan, Okan Atesal, Ahmet Toprak, Mehmet Deniz Caliskan, Ekmel Ozbay, "Disordered and densely packed ITO nanorods as an excellent lithography-free optical solar reflector metasurface for the radiative cooling of spacecraft," Proc. SPIE 11080, Metamaterials, Metadevices, and Metasystems 2019, 110801Q (5 September 2019); doi: 10.1117/12.2529003

SPIE Event: SPIE Nanoscience + Engineering, 2019, San Diego, California, United States 


\title{
Disordered and Densely Packed ITO Nanorods as an Excellent Lithography-Free Optical Solar Reflector Metasurface for the Radiative Cooling of Spacecraft
}

\author{
Deniz Umut YILDIRIM ${ }^{a, b, c}$, Amir Ghobadi ${ }^{a, b, c}$, Mahmut Can Soydan ${ }^{a, b, c}$, Okan Atesal ${ }^{a}$, \\ Ahmet Toprak $^{a}$, Mehmet Deniz Caliskan ${ }^{a}$, Ekmel Ozbay ${ }^{a, b, c, d}$ \\ ${ }^{a}$ NANOTAM-Nanotechnology Research Center, Bilkent University, 06800, Ankara, Turkey. \\ ${ }^{b}$ Department of Electrical and Electronics Engineering, Bilkent University, 06800, Ankara, \\ Turkey. \\ ${ }^{c}$ UNAM-Institute of Materials Science and Nanotechnology, Bilkent University, Ankara, \\ Turkey. \\ ${ }^{d}$ Department of Physics, Bilkent University, 06800, Ankara, Turkey.
}

\begin{abstract}
Optical Solar Reflectors (OSRs) form the physical interface between the spacecraft and space and they are essential for the stabilization and uniform distribution of temperature throughout the spacecraft. OSRs need to possess a spectrally selective response of broadband and perfect electromagnetic wave absorption in the thermalinfrared spectral range, while strongly reflecting the solar energy input. In this work, we experimentally show that disordered and densely packed ITO nanorod forests can be used as an excellent top-layer metasurface in a metal-insulator-oxide cavity configuration, and a thermal-emissivity of 0.97 is experimentally realized in the spectral range from 2.5 to $25 \mu \mathrm{m}$. The low-loss dielectric response of ITO in the solar spectrum, from $300 \mathrm{~nm}$ to $2.5 \mu \mathrm{m}$ range limited the solar absorptivity to an experimental value of 0.167 . These make our proposed design highly promising for its application in space missions due to combining high throughput, robustness, low cost with ultra-high performance.
\end{abstract}

Keywords: Metasurfaces, Oblique-angle deposition, Radiative Cooling, Optical solar reflectors, Transparent conductive oxides, Plasmonics

\section{Introduction}

Stabilization of temperature and its uniform distribution are crucial for spacecraft because most of their equipment become less reliable when operated outside of their acceptable temperature range. This, consequently, affect the success of the space missions adversely. Optical Solar Reflectors (OSRs) are secondary-surface mirrors that are coated to the external skin of spacecraft and they play a crucial role for the optimum performance of spacecraft and satellites during their missions. ${ }^{1-4}$ OSRs limit the solar energy input to the spacecraft while at the same time radiatively cooling it. OSRs then have to have a small absorption $\left(\alpha_{s}\right)$ of solar energy associated with the blackbody radiation of sun at $5778 \mathrm{~K}$, corresponding to the ultraviolet (UV), visible (VIS), and near-infrared (NIR) parts of the optical spectrum. Simultaneously, OSRs should strongly dissipate the heat generated on board by having a large thermal emissivity, and therefore broadband perfect absorption $\left(\epsilon_{I R}\right)$ in the mid-infrared (MIR) and far-infrared (FIR) parts of the spectrum (thermal-infrared) related to the blackbody radiation at $300 \mathrm{~K}$. Performance of an OSR can then be described by its Figure of Merit (FoM), $\epsilon_{I R} / \alpha_{s}$. The existing OSR solutions can be classified under two categories: conventional methods of using second-surface mirrors with quartz or teflon; ${ }^{1-3}$ and thin-film metamaterial based solutions. ${ }^{5-7}$

The conventional methods have extensively utilized quartz tiles or fluorinated ethylene propylene (FEP) films on top of Aluminum of Silver, to realize secondary surface mirrors. ${ }^{1-4}$ However, thickness of these films range

Further author information: (Send correspondence to D.U.Y. and E.O.)

D.U.Y.: E-mail: deniz.yildirim.ee@gmail.com

E.O.: E-mail: ozbay@bilkent.edu.tr

Metamaterials, Metadevices, and Metasystems 2019, edited by Nader Engheta,

Mikhail A. Noginov, Nikolay I. Zheludev, Proc. of SPIE Vol. 11080, 110801Q

(C) 2019 SPIE · CCC code: 0277-786X/19/\$21 - doi: 10.1117/12.2529003

Proc. of SPIE Vol. $11080110801 Q-1$ 
from a few hundreds of microns to millimeters, so they add huge launch and assembly cost to the spacecraft, because of the large weight. As a result, there is a strong opportunity of utilizing in metamaterial perfect absorbers to realize OSRs.

Metamaterials and their sub-wavelength thick counterparts, metasurfaces, represent a class of synthetic, man-made materials, whose sub-wavelength inclusions offer strong light-matter interactions. Their broad ${ }^{5-15}$ or narrow $^{16-24}$ resonant responses enable the efficient harvesting of the confined radiation by an absorbing layer such as metals, or semiconductors.

Transparent conductive Oxides (TCOs), such as Indium Tin Oxide (ITO), Aluminum-doped Zinc Oxide (AZO), and Gallium-doped Zinc Oxide (GZO) are a recently emerged class of materials ${ }^{25-31}$ with highly lossy plasmonic response in the infrared, which promises broad plasmonic resonances. Concurrently, their low-loss dielectric response in the solar spectrum would not accentuate solar absorption too much. Therefore, ultrabroadband perfect absorber devices that utilize TCOs are highly promising for the Optical Solar Reflector applications.

In realizing a perfect absorber, the designs in the literature predominantly made us of metal-insulator-metal cavities with top-layers requiring shapes that are patterned with electron beam lithography (EBL). ${ }^{32-46}$ Since OSRs are to coat the entire exterior surface of a spacecraft; their throughput, large scale compatibility and repeatability are as important as their performance. As a result, novel lithography-free designs are highly sought. Several methods to realize lithography-free perfect absorbers exist in the literature. Nevertheless, most methods either use planar lossy layers, ${ }^{47-50}$ where plasmonic resonances between nanounits does not exist, or slow chemical processes and/or large thermal budgets to realize randomness and disorder. ${ }^{51-55}$

In this work, we propose that disordered and densely packed ITO nanorod forests can be utilized as an excellent top-layer in a metal-insulator-oxide cavity configuration. These ITO nanorods are fabricated by utilizing oblique-angle deposition technique ${ }^{56-63}$ where the line-of-sight coating of PVD systems, in our case sputtering, and the shadowing effect resulted in the proposed top-layer, and the requirement for lithography is eliminated. Our devices achieved a record-high experimental thermal-emissivity of 0.97 in the spectral range from 2.5 to $25 \mu \mathrm{m}$, while the low-loss dielectric response of ITO in the solar spectrum, from $300 \mathrm{~nm}$ to $2.5 \mu \mathrm{m}$ range, also minimized the absorption losses, so the solar absorptivity is limited to an experimental value of 0.167. The layers have showed good adhesion and great uniformity over the entire wafer is verified as a proof-of-concept of large-scale compatibility of the design. Overall, our design is a great prototype for the applicability of thin-film metamaterial solutions to space missions.

\section{Results and Discussion}

\section{Theoretical and Numerical Design and Optimization of the OSR Metasurface}

Our proposed metasurface OSR is presented in in Fig. 1a. We name this device with the disordered and densely packed ITO nanorods as Device 1. This simple metal-insulator-oxide (MIO) cavity consists of a thick (125 nm) Aluminum (Al) back-reflector, to block the transmission channel, the spacer dielectric, $\mathrm{SiO}_{2}$, to create a cavity resonance, and the top absorbing layer, utilizing the ITO nanorods. The plasmonic response of ITO thermalinfrared, and the consequently excited localized surface plasmon resonances (LSPRs) are the main mechanisms in achieving broadband absorption in that range. The linewidth and the spectral position of these LSPRs are strongly linked to the the morphology (size, shape, spacing, and density) of the nanostructures. ${ }^{64}$ Therefore, to gain physical insight on how the nanorod morphology affects the spectral absorption from the solar spectrum to thermal-infrared, we analyze the same MIO cavity having a the top layer of periodic ITO discs. We name this testing device as Device 2, which is shown in Fig. 1b; and its the top view of its unit-cell is shown in Fig. 1c.

Device 2 is analyzed by utilizing finite-difference-time-domain (FDTD) simulations. We calculated the spectral absorption, $A(\lambda, T)$, of Device 2. $\epsilon_{I R}$ and $\alpha_{s}$ values are calculated, as shown in eq.2, using the $A(\lambda, T)$ and 

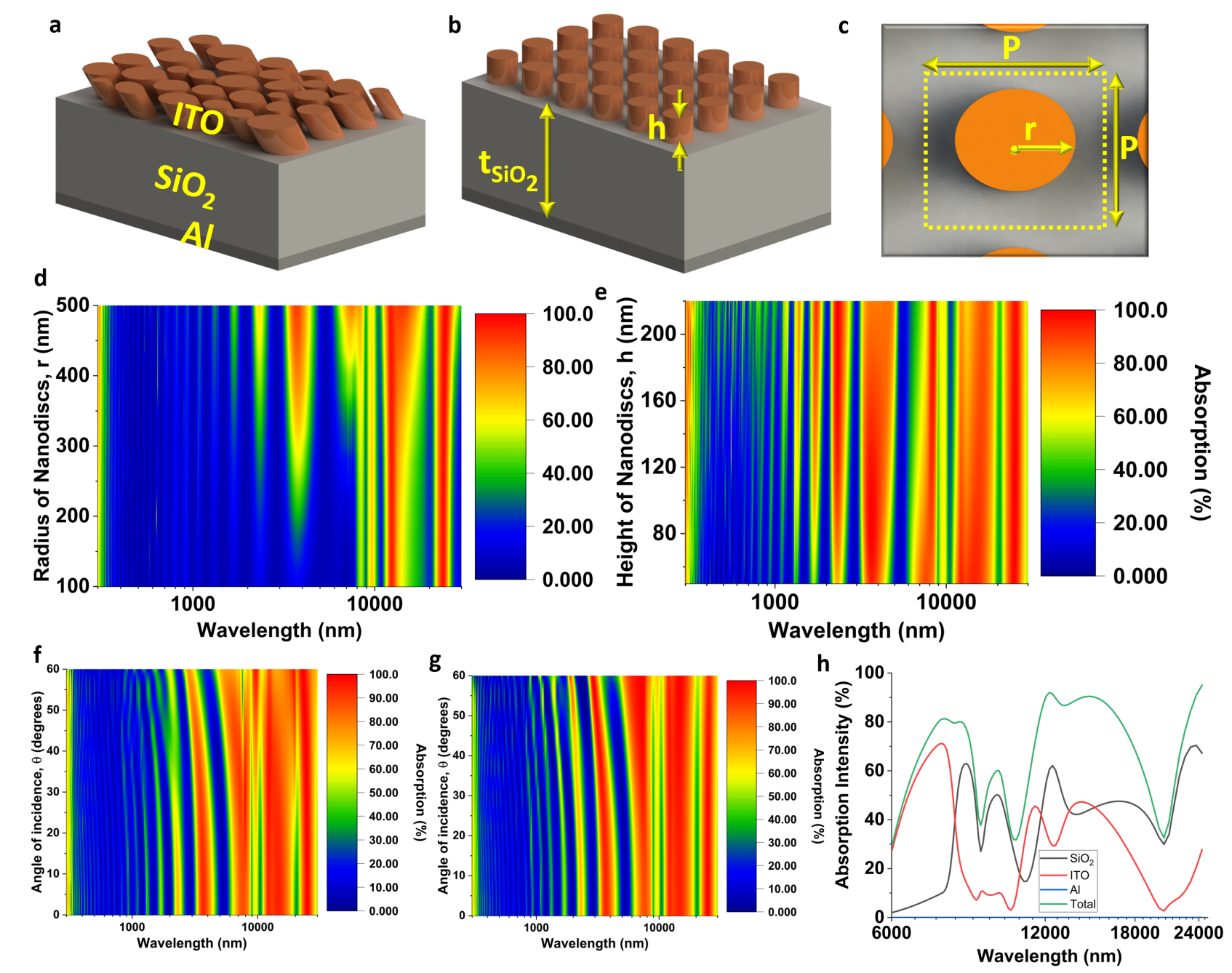

Figure 1: Schematics of Devices 1 and 2 and the parameter sweeps on the geometrical dimensions of Device 2. (a) Our proposed metasurface OSR, Device I. (b) Device II to investigate the effect of geometrical dimensions of a nanodisc on spectral absorption. (c) Top-view of Device II, showing the nanodisc radius and periodicity. Absorption spectrum of Device 2 under varying (d): Nanodisc radius, $r$, (e): Thickness of nanodiscs, $h$. Incidence angle dependent absorption spectrum under (f) p-polarized, (g) s-polarized EMW. (h): Contribution of each layer of Device 2 to the absorption in thermal-infrared.

the spectral radiance $B(\lambda, T)$, whose calculation is shown in eq.1.

$$
\begin{aligned}
B(\lambda, T) & =\frac{2 h c^{2}}{\lambda^{5}} \frac{1}{e^{\frac{h c}{\lambda k_{b} T}}-1} \\
\epsilon_{I R}, \alpha_{s} & =\frac{\int_{\lambda_{1}}^{\lambda_{2}} A(\lambda, T) B(\lambda, T) d \lambda}{\int_{\lambda_{1}}^{\lambda_{2}} B(\lambda, T) d \lambda}
\end{aligned}
$$

where $h, c$ and $k_{b}$ are Planck's constant, speed of light in vacuum and Boltzmann constant, respectively. The integrals are calculated from $300 \mathrm{~nm}$ to $2500 \mathrm{~nm}$ for the solar spectrum and from $2.5 \mu \mathrm{m}$ to $25 \mu \mathrm{m}$ for thermal infrared. $\lambda$ and $\mathrm{T}$ refers to the spectral wavelength and temperature, respectively. $\mathrm{T}$ takes the values of $5778 \mathrm{~K}$ for solar spectrum and $300 \mathrm{~K}$ for the thermal radiation. 
We first scrutinized the effect of the packing density of nanowires by sweeping $\mathrm{r}$, while $P, h$, and $t_{\mathrm{SiO}_{2}}$ are fixed at $1100 \mathrm{~nm}, 50 \mathrm{~nm}$, and $2000 \mathrm{~nm}$, respectively. Fig. 1d shows the spectral response of Device II for changing values of $r$. A broadband absorption spectrum is observed in thermal infrared due to the dominance of highloss plasmonic optical nature of ITO. Contrary to thermal infrared response, low-loss dielectric nature of ITO becomes dominant in solar spectrum and creates a Fabry-Pérot cavity, which causes oscillations in absorption spectrum. In the thermal-infrared, it is evident that as the nanodiscs become closer to each other, that is, as they get denser, and the coupling of dipole oscillations become stronger, ${ }^{65}$ and absorption spectrum becomes broader. In the solar spectrum, denser nanodiscs increase the fill factor of ITO, so the dielectric losses increase.

Next, we investigated the role of thickness of nanodiscs in the absorption spectrum. For this aim, we kept $P$, $r$ and $t_{\mathrm{SiO}_{2}}$ at $1100 \mathrm{~nm}, 500 \mathrm{~nm}$, and $2000 \mathrm{~nm}$, respectively; while changing $h$. Fig. 1f shows that, in the solar spectrum, thicker nanodiscs increase the light-matter interaction and the dielectric losses. In thermal-infrared, however, thicker nanodiscs cause less electromagnetic waves (EMWs) to reach to the cavity and weaken the cavity resonance, so the absorption strength is reduced.

Response of the metasurface to obliquely-incident radiation is also important when the application of this metasurface to space missions is considered. To examine this, we found the absorption spectrum of Device 2 under p- and s-polarized EMWs, incident at different angles, $\theta$, and the results are shown in Figures 1f,g. First, in the solar spectrum, the EMWs travel a longer path in the ITO layer, so $\alpha_{s}$ increases. Second, the cavity resonances are blue-shifted. This is because, in the case of normal-incidence, entire wavevector inside $\mathrm{SiO}_{2}$ contributes to the round-trip phase. However, when EMW is obliquely-incident, this phase contribution now comes from the real part of the axial component of complex wavevector inside $\mathrm{SiO}_{2}$, determined by Snell's Law. The resonance wavelength, $\lambda_{\text {res }}$ has to be reduced as the polar angle $\theta$ increses to satisfy the standing-wave condition in eq. $3{ }^{66}$

$$
2 m \pi=2\left(\frac{2 \pi}{\lambda_{\text {res }}}\right) n_{\mathrm{SiO}_{2}} 2 t_{\mathrm{SiO}_{2}} \cos (\theta)+\phi_{b}+\phi_{t}
$$

where $\phi_{b}+\phi_{t}$ are the phase terms owing to the Fresnel reflections at the top and bottom boundaries.

Overall, Device 2 is shown to support broad LSPRs in the thermal-infrared. With the calculated absorption spectra, Device 2 can reach an $\epsilon_{I R}$ of $0.8, \alpha_{s}$ of 0.18 , resulting in a moderate FoM of 4.5 at the expense of having a lithography step. The main limiting factor for Device 2 in achieving a higher FoM is its low $\epsilon_{I R}$, which is evident from Figs. 1d,e,f,g. Device II has a highly ordered ITO pattern, so its diffracting/scattering and EMW trapping features are poor. This causes a significant portion of the EMWs to be reflected by $\mathrm{SiO}_{2}$ at its strong phonon reflection (Reststrahlen) bands in thermal-infrared. The ITO metasurface with periodic nanodiscs cannot efficiently trap these radiation, and, consequently, they leave the device without being harnessed.

In the Reststrahlen bands, the EMW-ITO interaction is inherently limited and most of the absorption is due to absorption of the EMWs in the $\mathrm{SiO}_{2}$ layer. To verify this statement, we placed three three-dimensional (3D) monitors on the $\mathrm{Al}, \mathrm{SiO}_{2}$, and ITO layers, and found the contribution of each layer to absorption in the thermal-infrared, by using eq.4,

$$
\frac{d P_{\text {loss }}}{d V}=\frac{1}{2} w \epsilon^{\prime \prime}(w)|E|^{2}
$$

where $P_{\text {loss }}$ is the absorbed power, $V$ is the volume, $w$ is the angular frequency, $\epsilon^{\prime \prime}(w)$ is the imaginary part of the complex permittivity and $|E|^{2}$ is the modulus of the complex electric-field inside each layer. ${ }^{67}$ We integrated $d P_{\text {loss }} / d V$ over each layer and normalized to the incident power to obtain absorption of each. The absorption spectrum in Fig. $1 \mathrm{~h}$ clearly shows that in the phonon reflection bands (around 10 and $20 \mu \mathrm{m}$ ) of $\mathrm{SiO}_{2}$ harnessing of the EMWs reaching to the cavity is the main driver behind the absorption spectrum, while the contribution of ITO is minimal.

As a result, better trapping and harvesting of this reflected radiation while not increasing the solar absorption is of pivotal to achieve an FoM that is competitive to existing OSR solutions. 


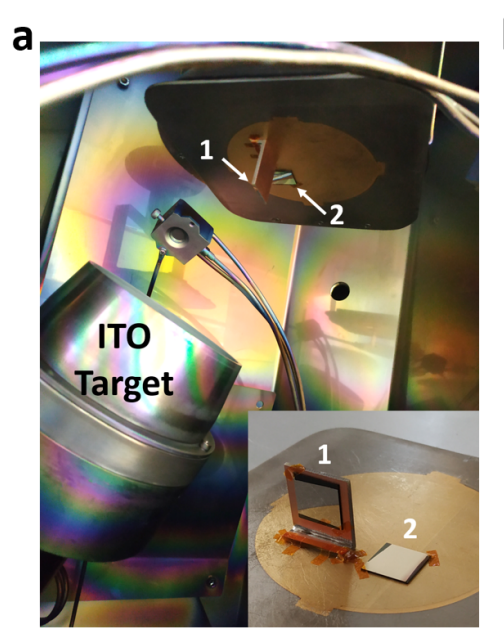

c

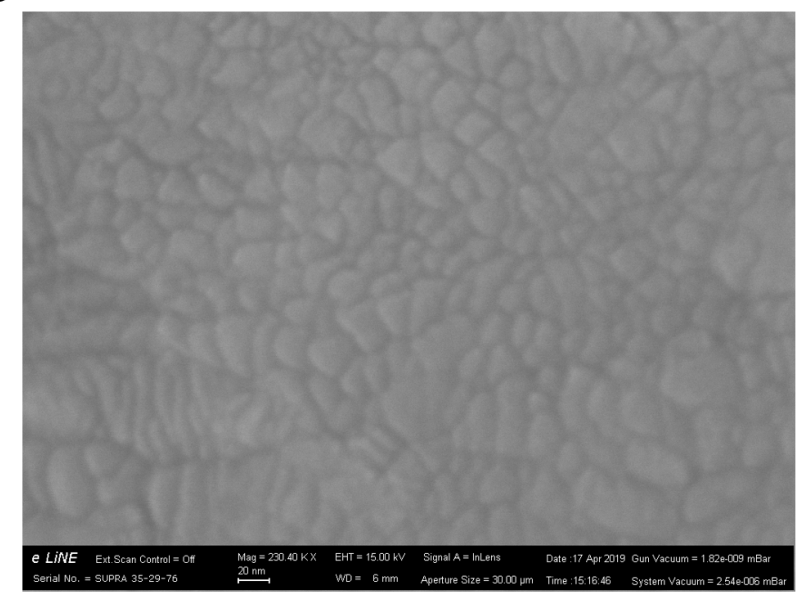

\section{b}
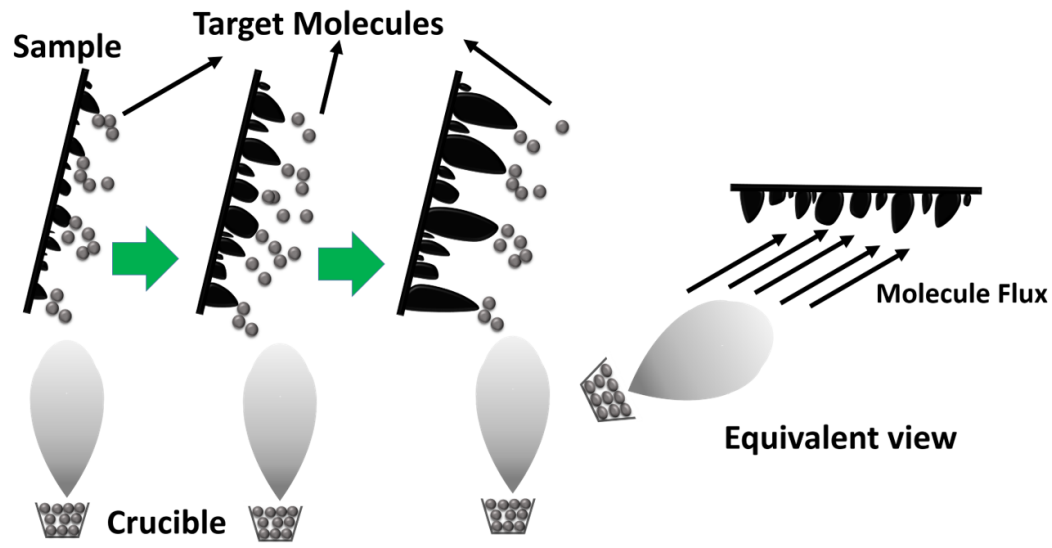
.

Equivalent view

d

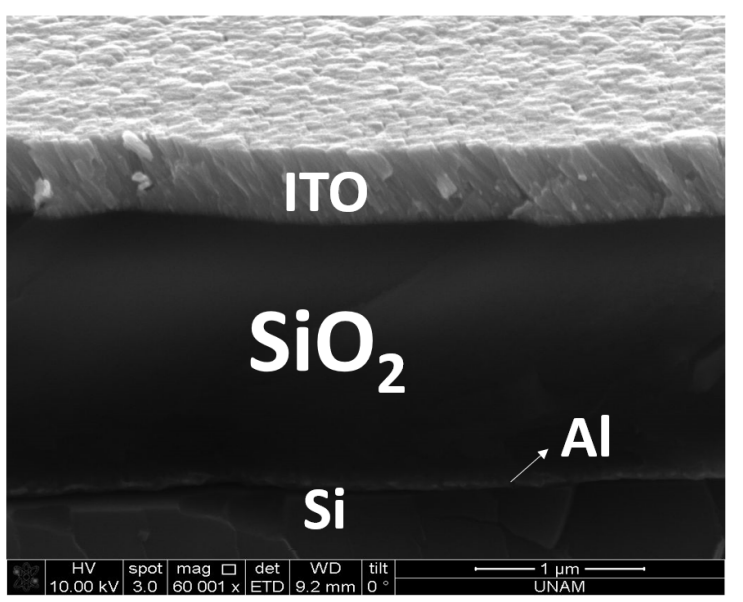

Figure 2: Fabrication of the metasurface OSR and the formation of ITO nanoforests. (a): Placement of samples in the sputtering chamber before coating of ITO, (b): Schematic illustration of line-of-sight coating in a sputtering system and the formation of tilted nanowires. (c): Top-view SEM micrographs of one of the fabricated devices. (d): cross-sectional SEM image a sample metasurface OSR.

\section{Fabrication and Characterization of the Metasurface OSR with Disordered and Densely Packed ITO Nanoforests as the Top-Layer}

In an effort to improve the performance of Device 2, by increasing $\epsilon_{I R}$ while keeping $\alpha_{s}$ at a reasonable value, we fabricated Device 1. To realize the top-layer metasurface with disordered and densely packed ITO nanorods, we employed the sputtering equipment but the sample is placed at an oblique-angle to the ITO target. This technique, known as oblique-angle deposition, utilizes the line-of-sight type coating of PVD systems with shadowing to fabricate nano-sized columnar fills with an intrinsic tilt and porosity. In Fig. 2a we show the placement of two samples in the sputtering system. While sample 1 is placed at an oblique angle to the ITO target, sample 2 is placed in an ordinary manner for planar deposition, which is used to compare its performance to Device 1 in the next part of the paper.

The process flow during the oblique-angle deposition and its equivalent picture is illustrated schematically in Fig. 2b. In this fabrication route, formation of random nucleation sites is occured at the intial stages of deposition. As deposition continues, primarily grown nucleation sites maintain coating on, while shadowed region behind them is not exposed to deposition. Consequently, the desired disordered and densely packed ITO 

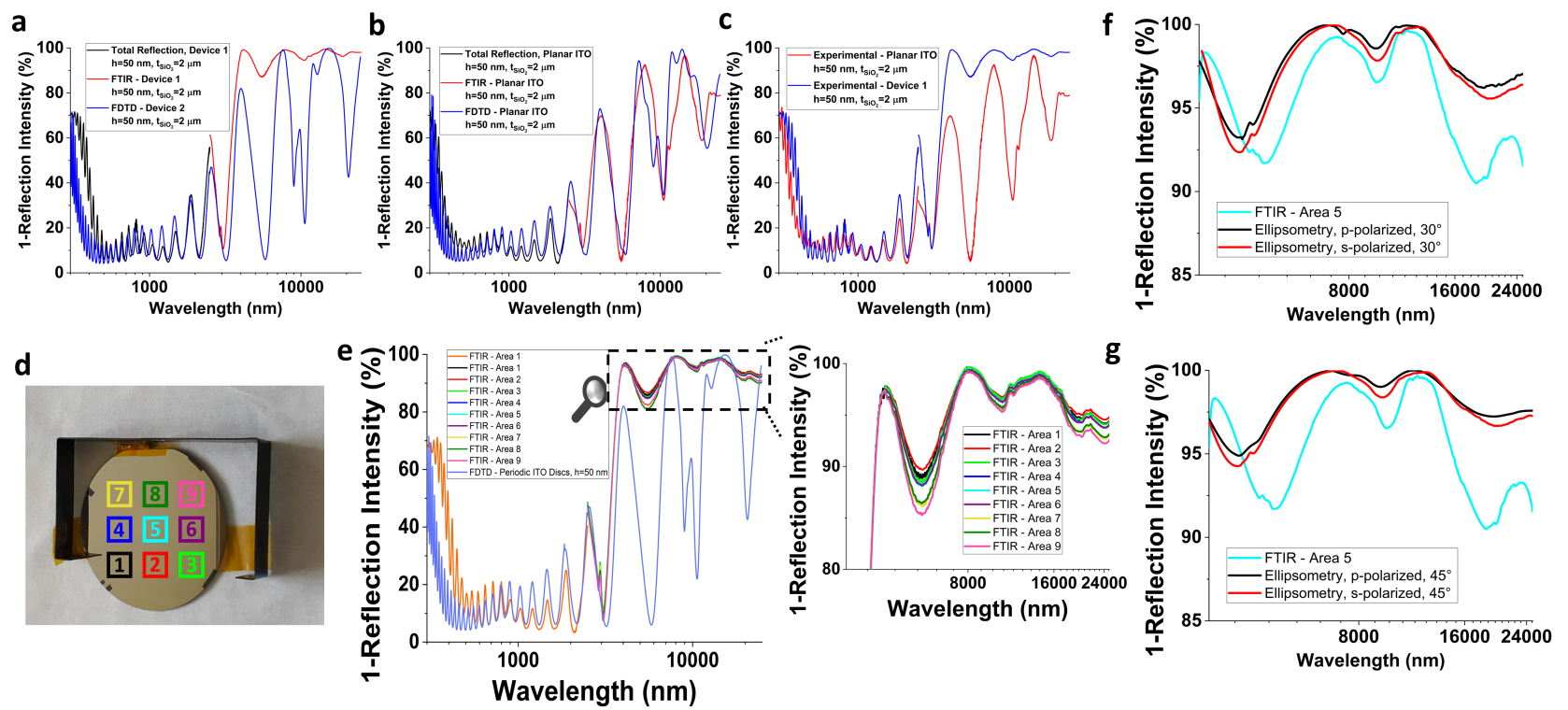

Figure 3: Optical Characterization of the fabricated small and large-area devices (a): Experimental absorption result for Device 1 with $h=50 \mathrm{~nm}$ and $t_{\mathrm{SiO}_{2}}=2 \mu \mathrm{m}$ compared to the numerical simulations of Device 2 with the same $h$ and $t_{\mathrm{SiO}_{2}}$, (b): Experimental absorption result for a planar device with $h=50 \mathrm{~nm}$ and $t_{\mathrm{SiO}_{2}}=$ $2 \mu \mathrm{m}$ compared to numerical simulations for same device, (c): Comparison of the absorption spectra of the planar device and Device 1 with $h=50 \mathrm{~nm}$ and $t_{\mathrm{SiO}_{2}}=2 \mu \mathrm{m}$. (d): Measurement areas in the fabricated wafers. (e): Experimental absorption result for Device 1 with $h=50 \mathrm{~nm}$ and $t_{\mathrm{SiO}_{2}}=2 \mu \mathrm{m}$. Absorption spectrum under obliquely-incident radiation at an incidence angle of (f): $30^{\circ}$, and $(\mathrm{g}): 45^{\circ}$

nanoforests are realized easily with a lithography-free process. They are intrinsically tilted towards the source due to deposition angle. Planar and cross-sectional scanning electron microscopy (SEM) images are shown in Figures 2c and d, respectively, and they verify the formation of such nanorod forests.

Reflection spectrum of the fabricated samples are measured separately in UV/VIS/NIR and thermal-infrared spectra. A total reflection spectrophotometer (Agilent Cary 5000) is employed in the UV/VIS/NIR ranges, while, Fourier Transform Infrared Spectroscopy (FTIR, Bruker- Vertex 70v, Hyperion microscope) technique is utilized in the thermal-infrared. The absorption spectrum for the fabricated devices are then found by $A=1-R$.

Initially, we fixed the the $\mathrm{SiO}_{2}$ thickness to its optimum value of $2 \mu \mathrm{m}$, while the thickness of the ITO layer is $50 \mathrm{~nm}$. The FDTD simulation results (for the case of periodic discs with $50 \mathrm{~nm}$ thickness, $1 \mu \mathrm{m}$ diameter and $1100 \mathrm{~nm}$ periodicity) were compared with the experimental absorption spectrum in Fig. 3a. It demonstrates a significant improvement for experimental results in terms of light-matter interaction, which yields ultra-broadband absorption spectrum in thermal infrared and an outstanding $\epsilon_{I R}$ value of 0.962 . However, it should be taken into consideration that high diffraction/scattering/light trapping design of the structure causes more interaction with the low-loss dielectric ITO, which may result higher $\alpha_{s}$. To find an optimum point, a thin ITO layer was chosen which provides a large $\epsilon_{I R}$ and restricts loss in the solar spectrum. Using $50 \mathrm{~nm}$ of ITO thickness, $\alpha_{s}$ was kept at 0.168 and FoM reached to a record-high value of 5.73.

To further demonstrate the power of the designed ITO nanoforests as a light-trapping scaffold, absorption spectrum of planar ITO layer and ITO nanoforest was compared. In Fig. 3b, FDTD simulation and experimental results for the planar ITO layer with $h=50 \mathrm{~nm}$ and $t_{\mathrm{SiO}_{2}}=2 \mu \mathrm{m}$ parameters are shown, and in Fig. 3c, experimental results of ITO nanoforest and planar ITO layer devices with the same parameters were compared. The planar ITO layer achieves $\alpha_{s}=0.151, \epsilon_{I R}=0.697$, and so, $F o M=4.61$. The worst $\epsilon_{I R}$ value is obtained for the planar ITO layer because it does not include any light-trapping capability and does not support excitation of LSPRs in Devices 1 and 2. However, diffraction/scattering effects are not present in planar design case; therefore, 
it does not suffer from excited guided-modes of the structure. ${ }^{68}$ Overall, ITO nanoforest design is shown to have better performance than planar ITO layer design.

Our proposed device is experimentally confirmed to provide an excellent scaffold for ultra-broadband thermalinfrared absorption and provides far superior $\epsilon_{I R}$ compared to Device 2. It achieves ultra-broadband perfect absorption in the thermal-infrared due to two primary reasons: 1) The disorder and the dense packing of the ITO nanorods resulted in excellent light-trapping capability by diffraction and scattering so the residing time of the thermal-radiation in the device is improved. This trapped radiation is then harnessed by the 2) hybrid system of ITO and Silicon Dioxide, where ITO contributes to the absorption by supporting multiple adjacent broad plasmonic resonances owing to the randomness in the morphology of the nanorods, and its highly lossy plasmonic behavior. The latter has strong phonon bands in the thermal-infrared, and therefore participates in the perfect absorption.

Since oblique-angle deposition does not use EBL, it enables large-scale compatibility of thin-film OSR devices. As a proof-of-concept, we fabricated Device 1 with $h=50 \mathrm{~nm}$ and $t_{\mathrm{SiO}_{2}}=2 \mu \mathrm{m}$ on a 4 -inch silicon wafer. The wafer is placed in the sputtering chamber at an angle to the source, similar to Fig. 2a. To examine the uniformity of the fabrication, the wafer is characterized from 9 different areas, which are shown in Fig. 3d. Experimentally obtained absorption spectra for different areas are shown in Fig. 3e. Solar spectrum absorption is measured only from Area 5 and $\alpha_{s}$ is calculated as 0.167. Spectral response in thermal-infrared is measured from all 9 areas, and we zoomed into $3 \mu \mathrm{m}$ to $25 \mu$ spectral range to further demonstrate the variations in the absorption spectra between different areas. We found that the smallest $\epsilon_{I R}$ is 0.957 , while its highest value reached 0.97 , so the variations within the wafer is not significant.

Reflection spectrum of the fabricated Device 1, which has parameters of $h=50 \mathrm{~nm}$ and $t_{\mathrm{SiO}_{2}}=1.55 \mu \mathrm{m}$, under oblique angle radiation was measured from $3 \mu \mathrm{m}$ to $25 \mu \mathrm{m}$ by using ellipsometry technique (J.A. Woollam Co. Inc. IR-VASE Mark II ellipsometer). s-polarized and p-polarized electromagnetic waves were impinged on the sample with 30 and 45 incidence angles, and the resultant spectra are shown in Figs. 3f and 3g, respectively. A blue-shift in the spectral response and an increase in absorption is observed, similar to numerical results for Device 2, as in Figs. 1f and g.

We have clearly revealed the extraordinary performance and compatibility of our proposed device for OSR applications due to having very high $\epsilon_{I R}$ and a small $\alpha_{s}$. It is also large-scale compatible and uniform, and its thickness is at least two orders of magnitude smaller than that of the conventional OSRs. Further studies in finding the heat transfer and the cooling rates are needed for the eventual application of these devices to actual space missions. Moreover, new ideas can be developed such as flexible substrates to improve applicability. In addition, the presented results are very promising in application areas of thermal-imaging with labeled-security, and daytime radiative coolers. ${ }^{12,13,69}$ However, adaptation of the overall absorption response of the device over thermal infrared region to the atmospheric absorption lines should be fulfilled, which is an present ongoing study.

\section{Conclusions}

In conclusion, we numerically and experimentally analyzed metasurface optical solar reflector devices that utilize disordered and densely packed ITO nanorod forests as the top absorbing layer. The fabricated devices reached a record-high thermal-emissivity of 0.968 , and Figure of Merit of 5.73. The adoption of oblique-angle deposition technique alse enabled large-scale and high-throughput fabrication. The experimental results of the proposed devices are compared with the numerical results for periodic ITO discs and the experimental spectrum for planar ITO thin-film, as the top absorbing layer. These comparisons further indicated the absorption enhancement by the ITO nanoforests, which is mainly due to increasing the light-matter interactions in the strong phonon reflection bands of $\mathrm{SiO}_{2}$. Overall, our ultra-high performance, yet large-scale compatible devices are highly promising for the radiative cooling of spacecraft during their space missions, and it is also highly applicable to many areas such as terrestrial radiative coolers and thermal imaging for labeled security purposes.

\section{Acknowledgements}

Authors acknowledge financial support from Scientific and Technological Research Council of Turkey (TUBITAK) and DPT-HAMIT under the Project Nos. 113E331, 114E374, and 115F560. One of the authors (E.O.) also acknowledges partial support from the Turkish Academy of Sciences (TUBA). 


\section{REFERENCES}

[1] Pisacane, V., [Fundamentals of Space Systems] (2005).

[2] Ovchinnikov, M., Mckenna-lawlor, S., Psychology, S., Kanas, N., Manuy, D., Larson, W. J., and Wertz, J. R., [Space Mission Analysis and Design] (1991).

[3] Gilmore, D., [Spacecraft Thermal Control Handbook, Volume I: Fundamental Technologies], American Institute of Aeronautics and Astronautics, Inc. (dec 2002).

[4] BREUCH, R. A. and MARSHALL, K. N., "Optical solar reflector - A highly stable, low alpha sub S/epsilon spacecraft thermal control surface.," Journal of Spacecraft and Rockets 5, 1051-1056 (sep 1968).

[5] Yildirim, D. U., Ghobadi, A., Soydan, M. C., Atesal, O., Toprak, A., Caliskan, M. D., and Ozbay, E., "Disordered and Densely Packed ITO Nanorods as an Excellent Lithography-Free Optical Solar Reflector Metasurface," ACS Photonics (2019).

[6] Sun, K., Riedel, C. A., Wang, Y., Urbani, A., Simeoni, M., Mengali, S., Zalkovskij, M., Bilenberg, B., De Groot, C. H., and Muskens, O. L., "Metasurface Optical Solar Reflectors Using AZO Transparent Conducting Oxides for Radiative Cooling of Spacecraft," ACS Photonics 5(2), 495-501 (2018).

[7] Sun, K., Riedel, C. A., Urbani, A., Simeoni, M., Mengali, S., Zalkovskij, M., Bilenberg, B., De Groot, C. H., and Muskens, O. L., "VO2 Thermochromic Metamaterial-Based Smart Optical Solar Reflector," ACS Photonics 5(6), 2280-2286 (2018).

[8] Rephaeli, E. and Fan, S., "Absorber and emitter for solar thermo-photovoltaic systems to achieve efficiency exceeding the Shockley-Queisser limit," Optics Express (2009).

[9] Tong, J. K., Hsu, W. C., Huang, Y., Boriskina, S. V., and Chen, G., "Thin-film 'thermal well' emitters and absorbers for high-efficiency thermophotovoltaics," Scientific Reports (2015).

[10] García De Arquer, F. P., Mihi, A., and Konstantatos, G., "Large-Area Plasmonic-Crystal-Hot-ElectronBased Photodetectors," ACS Photonics (2015).

[11] Li, W. and Valentine, J., "Metamaterial perfect absorber based hot electron photodetection," Nano Letters (2014).

[12] Lu, Y., Chen, Z., Ai, L., Zhang, X., Zhang, J., Li, J., Wang, W., Tan, R., Dai, N., and Song, W., "A Universal Route to Realize Radiative Cooling and Light Management in Photovoltaic Modules," Solar $R R L$ 1700084, 1700084 (2017).

[13] Ko, B., Lee, D., Badloe, T., and Rho, J., "Metamaterial-Based Radiative Cooling: Towards Energy-Free All-Day Cooling," Energies 12(1), 89 (2018).

[14] Zhao, B., Hu, M., Ao, X., Chen, N., and Pei, G., "Radiative cooling: A review of fundamentals, materials, applications, and prospects," Applied Energy 236(December 2018), 489-513 (2019).

[15] Soydan, M. C., Ghobadi, A., Yildirim, D. U., Erturk, V. B., and Ozbay, E., "All Ceramic-Based Metal-Free Ultra-broadband Perfect Absorber," Plasmonics (2019).

[16] Ellenbogen, T., Seo, K., and Crozier, K. B., "Chromatic plasmonic polarizers for active visible color filtering and polarimetry," Nano Letters (2012).

[17] Landy, N. I., Bingham, C. M., Tyler, T., Jokerst, N., Smith, D. R., and Padilla, W. J., "Design, theory, and measurement of a polarization-insensitive absorber for terahertz imaging," Physical Review B - Condensed Matter and Materials Physics (2009).

[18] Offermans, P., Schaafsma, M. C., Rodriguez, S. R., Zhang, Y., Crego-Calama, M., Brongersma, S. H., and Gómez Rivas, J., "Universal scaling of the figure of merit of plasmonic sensors," ACS Nano (2011).

[19] Stewart, M. E., Anderton, C. R., Thompson, L. B., Maria, J., Gray, S. K., Rogers, J. A., and Nuzzo, R. G., "Nanostructured plasmonic sensors," (2008).

[20] Tittl, A., Michel, A. K. U., Schäferling, M., Yin, X., Gholipour, B., Cui, L., Wuttig, M., Taubner, T., Neubrech, F., and Giessen, H., "A Switchable Mid-Infrared Plasmonic Perfect Absorber with Multispectral Thermal Imaging Capability," Advanced Materials (2015).

[21] Xu, T., Wu, Y.-K., Luo, X., and Guo, L. J., "Plasmonic nanoresonators for high-resolution colour filtering and spectral imaging," Nature Communications (2010).

[22] Yildirim, D. U., Ghobadi, A., and Ozbay, E., "Near-absolute polarization insensitivity in grapheme based ultra-narrowband perfect visible light absorber," Scientific Reports 8(1), 1-14 (2018). 
[23] Yokogawa, S., Burgos, S. P., and Atwater, H. A., "Plasmonic color filters for CMOS image sensor applications," Nano Letters (2012).

[24] Yildirim, D. U., Ghobadi, A., Soydan, M. C., Gokbayrak, M., Toprak, A., Butun, B., and Ozbay, E., "Colorimetric and Near-Absolute Polarization Insensitive Refractive-Index Sensing in All-Dielectric GuidedMode Resonance based Metasurface," The Journal of Physical Chemistry C (2019).

[25] Naik, G. V., Shalaev, V. M., and Boltasseva, A., "Alternative plasmonic materials: Beyond gold and silver," (2013).

[26] Withnall, R., [Encyclopedia of Modern Optics] (2005).

[27] Li, S. Q., Guo, P., Zhang, L., Zhou, W., Odom, T. W., Seideman, T., Ketterson, J. B., and Chang, R. P. H., "Infrared plasmonics with indium-tin-oxide nanorod arrays," ACS Nano (2011).

[28] Khurgin, J. B., "Replacing noble metals with alternative materials in plasmonics and metamaterials: How good an idea?," in [Philosophical Transactions of the Royal Society A: Mathematical, Physical and Engineering Sciences], (2017).

[29] Kim, J., Dutta, A., Naik, G. V., Giles, A. J., Bezares, F. J., Ellis, C. T., Tischler, J. G., Mahmoud, A. M., Caglayan, H., Glembocki, O. J., Kildishev, A. V., Caldwell, J. D., Boltasseva, A., and Engheta, N., "Role of epsilon-near-zero substrates in the optical response of plasmonic antennas," Optica (2016).

[30] Naik, G. V. and Boltasseva, A., "Semiconductors for plasmonics and metamaterials," Physica Status Solidi - Rapid Research Letters (2010).

[31] Abb, M., Wang, Y., Papasimakis, N., De Groot, C. H., and Muskens, O. L., "Surface-enhanced infrared spectroscopy using metal oxide plasmonic antenna arrays," Nano Letters (2014).

[32] Aydin, K., Ferry, V. E., Briggs, R. M., and Atwater, H. A., "Broadband polarization-independent resonant light absorption using ultrathin plasmonic super absorbers," Nature Communications (2011).

[33] Liang, Q., Yu, W., Zhao, W., Wang, T., Zhao, J., Zhang, H., and Tao, S., "Numerical study of the metananopyramid array as efficient solar energy absorber," Optical Materials Express (2013).

[34] Ding, F., Dai, J., Chen, Y., Zhu, J., Jin, Y., and Bozhevolnyi, S. I., "Broadband near-infrared metamaterial absorbers utilizing highly lossy metals," Scientific Reports (2016).

[35] Nielsen, M. G., Pors, A., Albrektsen, O., and Bozhevolnyi, S. I., "Efficient absorption of visible radiation by gap plasmon resonators," Optics Express (2012).

[36] Liang, Q., Wang, T., Lu, Z., Sun, Q., Fu, Y., and Yu, W., "Metamaterial-Based Two Dimensional Plasmonic Subwavelength Structures Offer the Broadest Waveband Light Harvesting," Advanced Optical Materials (2013).

[37] Feng, Q., Pu, M., Hu, C., and Luo, X., "Engineering the dispersion of metamaterial surface for broadband infrared absorption," Optics Letters (2012).

[38] Guo, W., Liu, Y., and Han, T., "Ultra-broadband infrared metasurface absorber," Optics Express (2016).

[39] Wang, H. and Wang, L., "Perfect selective metamaterial solar absorbers," Optics Express (2013).

[40] Bossard, J. A., Lin, L., Yun, S., Liu, L., Werner, D. H., and Mayer, T. S., "Near-ideal optical metamaterial absorbers with super-octave bandwidth," ACS Nano (2014).

[41] Ghobadi, A., Hajian, H., Gokbayrak, M., Abedini Dereshgi, S., Toprak, A., Butun, B., and Ozbay, E., "Visible light nearly perfect absorber : an optimum unit cell arrangement for near absolute polarization insensitivity," Optics Express (2017).

[42] Ding, F., Jin, Y., Li, B., Cheng, H., Mo, L., and He, S., "Ultrabroadband strong light absorption based on thin multilayered metamaterials," Laser and Photonics Reviews (2014).

[43] Cui, Y., Fung, K. H., Xu, J., Ma, H., Jin, Y., He, S., and Fang, N. X., "Ultrabroadband light absorption by a sawtooth anisotropic metamaterial slab," Nano Letters (2012).

[44] Zhu, J., Ma, Z., Sun, W., Ding, F., He, Q., Zhou, L., and Ma, Y., "Ultra-broadband terahertz metamaterial absorber," Applied Physics Letters (2014).

[45] Long, C., Yin, S., Wang, W., Li, W., Zhu, J., and Guan, J., "Broadening the absorption bandwidth of metamaterial absorbers by transverse magnetic harmonics of 210 mode," Scientific Reports (2016).

[46] He, X., Yan, S., Lu, G., Zhang, Q., Wu, F., and Jiang, J., "An ultra-broadband polarization-independent perfect absorber for the solar spectrum," RSC Advances (2015). 
[47] Ghobadi, A., Hajian, H., Butun, B., and Ozbay, E., "Strong Light-Matter Interaction in Lithography-Free Planar Metamaterial Perfect Absorbers," ACS Photonics 5, 4203-4221 (2018).

[48] Li, Z., Butun, S., and Aydin, K., "Large-area, Lithography-free super absorbers and color filters at visible frequencies using ultrathin metallic films," ACS Photonics (2015).

[49] Abedini Dereshgi, S., Ghobadi, A., Hajian, H., Butun, B., and Ozbay, E., "Ultra-Broadband, LithographyFree, and Large-Scale Compatible Perfect Absorbers: The Optimum Choice of Metal layers in MetalInsulator Multilayer Stacks," Scientific Reports (2017).

[50] Ding, F., Mo, L., Zhu, J., and He, S., "Lithography-free, broadband, omnidirectional, and polarizationinsensitive thin optical absorber," Applied Physics Letters (2015).

[51] Ghobadi, A., Dereshgi, S. A., Hajian, H., Birant, G., Butun, B., Bek, A., and Ozbay, E., "97 percent light absorption in an ultrabroadband frequency range utilizing an ultrathin metal layer: Randomly oriented, densely packed dielectric nanowires as an excellent light trapping scaffold," Nanoscale 9(43), 16652-16660 (2017).

[52] Ghobadi, A., Dereshgi, S. A., Hajian, H., Bozok, B., Butun, B., and Ozbay, E., "Ultra-broadband, wide angle absorber utilizing metal insulator multilayers stack with a multi-thickness metal surface texture," Scientific Reports (2017).

[53] Ghobadi, A., Hajian, H., Dereshgi, S. A., Bozok, B., Butun, B., and Ozbay, E., "Disordered Nanohole Patterns in Metal-Insulator Multilayer for Ultra-broadband Light Absorption: Atomic Layer Deposition for Lithography Free Highly repeatable Large Scale Multilayer Growth," Scientific Reports (2017).

[54] Ghobadi, A., Hajian, H., Rashed, A. R., Butun, B., and Ozbay, E., "Tuning the metal filling fraction in absorbers to maximize the absorption bandwidth," Photonics Reseach (2018).

[55] Bae, K., Kang, G., Cho, S. K., Park, W., Kim, K., and Padilla, W. J., "Flexible thin-film black gold membranes with ultrabroadband plasmonic nanofocusing for efficient solar vapour generation," Nature Communications (2015).

[56] Barranco, A., Borras, A., Gonzalez-Elipe, A. R., and Palmero, A., "Perspectives on oblique angle deposition of thin films: From fundamentals to devices," (2016).

[57] Wang, Z., West, P. R., Meng, X., Kinsey, N., Shalaev, V. M., and Boltasseva, A., "Angled physical vapor deposition techniques for non-conformal thin films and three-dimensional structures," MRS Communications 6(1), 17-22 (2016).

[58] Grüner, C., Liedtke, S., Bauer, J., Mayr, S. G., and Rauschenbach, B., "Morphology of Thin Films Formed by Oblique Physical Vapor Deposition," ACS Applied Nano Materials 1(3), 1370-1376 (2018).

[59] Mark, A. G., Gibbs, J. G., Lee, T. C., and Fischer, P., "Hybrid nanocolloids with programmed threedimensional shape and material composition," Nature Materials (2013).

[60] Jensen, M. and Brett, M. J., "Square spiral 3D photonic bandgap crystals at telecommunications frequencies.," Optics express (2005).

[61] Lintymer, J., Gavoille, J., Martin, N., and Takadoum, J., "Glancing angle deposition to modify microstructure and properties of sputter deposited chromium thin films," Surface and Coatings Technology (2003).

[62] Xi, J. Q., Schubert, M. F., Kim, J. K., Schubert, E. F., Chen, M., Lin, S. Y., Liu, W., and Smart, J. A., "Optical thin-film materials with low refractive index for broadband elimination of Fresnel reflection," Nature Photonics (2007).

[63] Xiao, X., Dong, G., Shao, J., He, H., and Fan, Z., "Optical and electrical properties of SnO2:Sb thin films deposited by oblique angle deposition," Applied Surface Science (2010).

[64] Maier, S. A., [Plasmonics: Fundamentals and applications] (2007).

[65] Abb, M., Wang, Y., Papasimakis, N., De Groot, C. H., and Muskens, O. L., "Surface-enhanced infrared spectroscopy using metal oxide plasmonic antenna arrays," Nano Letters 14(1), 346-352 (2014).

[66] Shabahang, S., Kondakci, H. E., Villinger, M. L., Perlstein, J. D., El Halawany, A., and Abouraddy, A. F., "Omni-resonant optical micro-cavity," Scientific Reports (2017).

[67] Balabanian, N., "Impedance Matching," IRE Transactions on Microwave Theory and Techniques 3(4), 53 (1955).

[68] Fan, S. and Joannopoulos, J. D., "Analysis of guided resonances in photonic crystal slabs," Physical Review $B$ (2002).

[69] Zhai, Y., Ma, Y., David, S. N., Zhao, D., Lou, R., Tan, G., Yang, R., and Yin, X., "Scalable-manufactured randomized glass-polymer hybrid metamaterial for daytime radiative cooling," Science (2017). 